\title{
Management of thoracic spine dislocation by total vertebrectomy and spine shortening: case report
}

\author{
Sami I. AlEissa ${ }^{1} \cdot$ Faisal M. Konbaz $\mathbb{1}^{1}{ }^{1} \cdot$ Turki S. AlMugren $^{1}{ }^{1} \cdot$ Fahad H. AlHelal $^{1} \cdot$ Majed S. Abalkhail $^{1}$ \\ Adnan A. AlKandari ${ }^{1} \cdot$ Mohammed T. AlZahrani ${ }^{1}$
}

Received: 5 June 2020 / Revised: 29 July 2020 / Accepted: 3 August 2020

(C) International Spinal Cord Society 2020

\begin{abstract}
Introduction The treatment of thoracic spine fracture-dislocations is now well established with the recent progress in spine surgery. Although most affected individuals have a degree of spinal cord injury (SCI), early surgical reduction, and stabilization of the unstable deformity allow an immediate program of rehabilitation. Vertebrectomy is considered as the last surgical technique reserved for the most persistent spinal deformities that cannot be brought to an acceptable correction with less invasive methods.

Case presentation We present a case of a 19-year-old male with a sub-acute thoracic spine fracture-dislocation at the level of T7-T8 who underwent a posterior T8 vertebrectomy with reduction and instrumentation from T4 down to T1. The individual had excellent results during follow-ups regarding alignment, fusion, and rehab program.

Discussion In this case, we present the good outcome of vertebrectomy and spine shortening in an individual with thoracic spine fracture-dislocation, and the advantages of posterior approach.
\end{abstract}

\section{Introduction}

The treatment of thoracic spine fracture-dislocations is now well established with recent progress in spine surgery. Although most of the individuals have a degree of spinal cord injury (SCI), early surgical reduction, and stabilization of unstable deformity allows an immediate program of rehabilitation [1]. Vertebrectomy is considered as the last surgical technique reserved for the most persistent spinal deformities that cannot be brought to an acceptable correction with less invasive methods [2]. It was first illustrated in 1922 by Mac-Lennan, who described an apical resection from a posterior-only approach with post-operative casting for the treatment of severe congenital scoliosis [3]. His technique was used later in the management of oncological, infectious, and traumatic disorders [4-6].

In 2004, Barbera reported a case of a young paraplegic individual, presenting with painful kyphosis and a chronic

Turki S. AlMugren

turki.almugren@gmail.com

1 Department of Surgery, King Abdul-aziz Medical City, Riyadh, Saudi Arabia dislocation of T12-L1 [7]. The first reported case of an acute vertebrectomy of a thoracic spine dislocation was by Obeid et al., followed by Barcelos and Botelho [8, 9]. To our knowledge, surgical management of thoracic dislocation by acute complete vertebrectomy has only been reported two times in the literature. The purpose of this case is to present the surgical steps, safety and efficacy of acute vertebrectomy in a thoracic spine dislocation due to high energy trauma in a young individual.

\section{Case presentation}

A 19-year-old male with no significant past medical or surgical history, involved in a motor vehicle accident 23 days prior, presented to our hospital. He was a front seat passenger not wearing a seatbelt. Moreover, the car rolled over multiple times and he was ejected from the car. He was transferred by ambulance to a peripheral hospital that managed his life-threatening injuries, including bilateral hemothorax, spleen, and liver lacerations. Three weeks after his injury, he was shifted to our hospital for the management of his thoracic spine fracture-dislocation.

Upon admission, he was stable with a Glasgow Coma Scale of 15. His examination showed a T6 AIS A SCI with 
Fig. 1 a, b Axial and sagittal cuts of the thoracic spine computed tomography showing spondyloptosis and complete dislocation of $\mathrm{T} 7$ anterior to $\mathrm{T} 8$ with overlap.
A

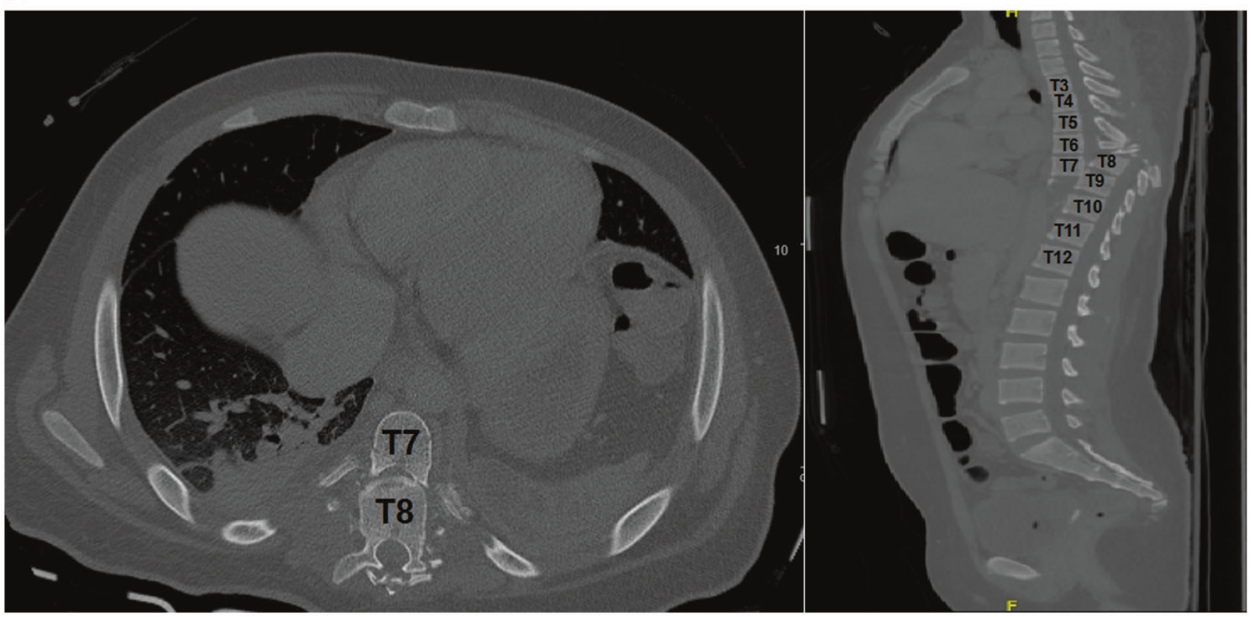

complete absence of sensation and motor function in both lower extremities and no anal sensation or voluntary contraction. Computed tomography of the spine showed spondyloptosis and complete dislocation of $\mathrm{T} 7$ anterior to T8 with overlap (Fig. 1a, b). Bilateral Doppler ultrasonography of the lower limbs showed thrombosis in the right popliteal and left femoral veins. The individual was scheduled for T8 vertebrectomy with reduction and instrumentation from $\mathrm{T} 4$ down to $\mathrm{T} 11$ by the spine team after treatment with anticoagulation medication and insertion of a temporary prophylactic inferior vena cava.

In the operating room, after general anesthesia induction and one dose of prophylactic antibiotic, the individual was shifted to a prone position. After prepping and draping of the back, we started our skin incision posteriorly through midline over the thoracic spine to expose the spinous process followed by subperiosteal dissection of the paraspinal muscles to the tips of transverse processes. Exposure was performed from T4 to T11 and confirmed by fluoroscopy. There was a complete dislocation and a marked kyphotic deformity at the level of T7-T8, and the body of the T8 vertebra was behind the body of T7. Pedicle screws were inserted under fluoroscopy guidance from the T4 to T11 vertebrae (Fig. 2b). No screws were inserted in the T8 vertebra as it was planned for vertebrectomy. After laminectomy of the T8-T9 vertebrae was performed, the spinal cord was exposed and found to be severely injured and contused with a fibrous formation over the dura matter and no CSF leak. We did not attempt to remove this fibrous tissue to avoid injuring the dura and cause leakage. We resected the head of the 8 th rib bilaterally, followed by ligation of the bilateral nerve roots of $\mathrm{T} 8$. After that, discectomy of T8-T9 was completely performed, and then by using an osteotome the vertebrectomy was performed as en bloc (Fig. 2c). A rod distractor was utilized to distract and overcome the overlap between $\mathrm{T} 7$ and $\mathrm{T} 9$ vertebrae, and after maintaining proper distraction, a Cobb dissector was inserted in the fracture gap to lever the T7 vertebra proximally (Fig. 2d). Refreshing the upper endplate of T9 and lower endplate of $\mathrm{T} 7$ was performed to enhance the fusion. Two body screws in T7 were inserted under fluoroscopy as there was no pedicle in the fractured vertebra, and the reduction was achieved by using reduction screws for the proximal segment vertebrae (T4-T7). Two cobalt chrome rods were applied and final AP and lateral X-rays were satisfying and showed excellent realignment of the spine (Figs. 2e and 3). After irrigation with normal saline, decortication of the transverse processes and laminae throughout the instrumented levels was performed alone with closure in layers.

On the first post-operative day, there was no change in physical examination and the individual was started on a bedside physiotherapy and occupational therapy. Two weeks after the surgery, clips and inferior vena cava filter were removed, and CT of the thoracic spine was done (Fig. 4). After that, individual was shifted to a rehab ward for an extensive rehab program. Once the individual completed his inpatient traumatic SCI protocol and discharged, he came via a wheelchair to the clinic on his scheduled appointment 6 months after surgery with unchanged neurological examination, and $\mathrm{X}$-rays showed good alignment and stable fixation (Fig. 5a, b). He was lost to follow-up after 6 months as he was planning to go to a rehab center outside the country.

\section{Discussion}

Thoracic spine fracture-dislocations are often associated with SCI. Due to the inherited mechanical stability of the thoracic spine, as a result of the sagittal facets orientation and the presence of the costovertebral articulation, high 
Fig. 2 Three dimension graphic illustration of total vertebrectomy and spine shortening. a Thoracic spine dislocation at T7-T8. b Screws inserted in levels above and below. c Vertebrectomy of T8 and insertion of body screws in T7. d, e Rods distraction and application after full reduction of the accomplished.

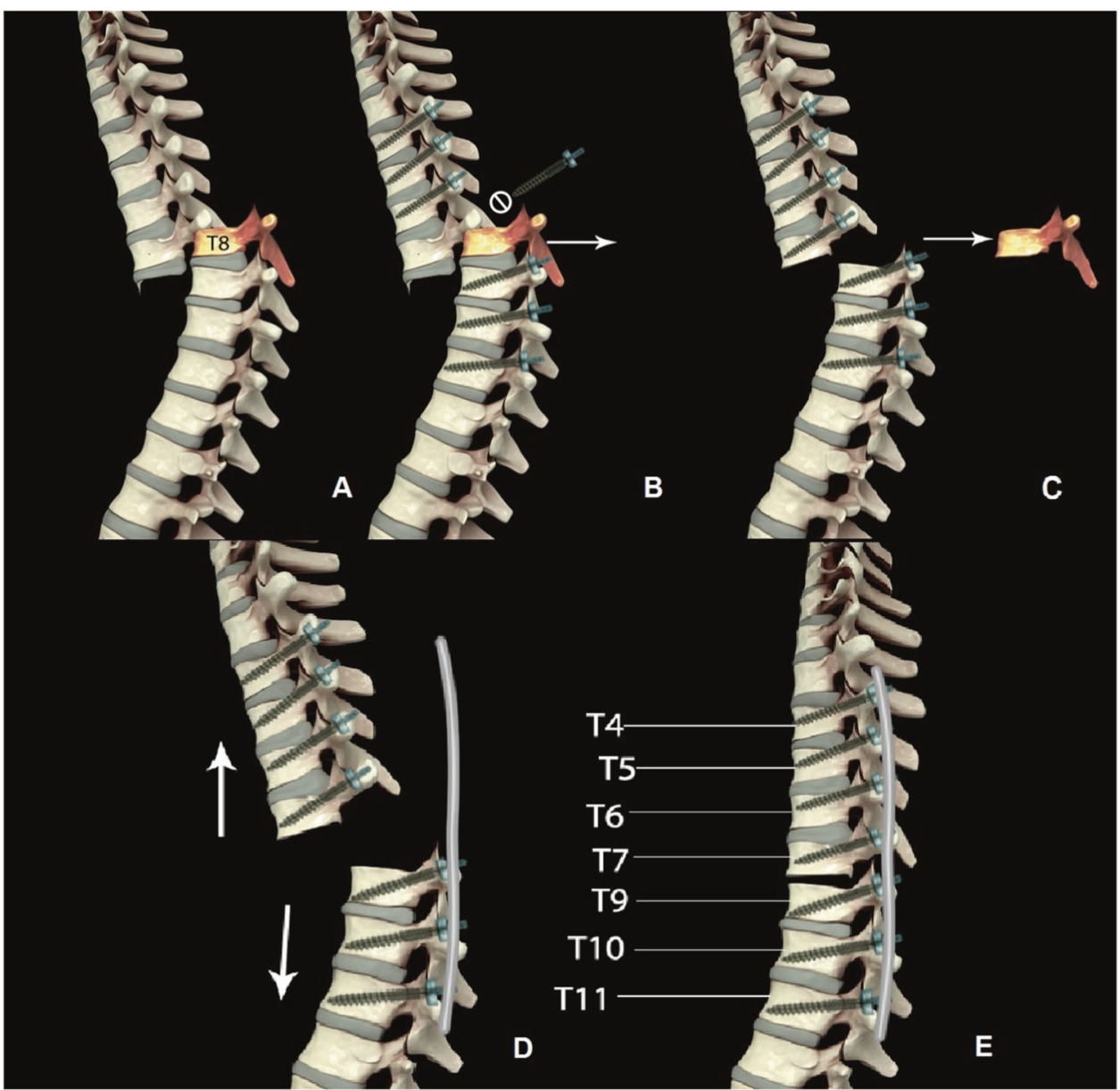

energy trauma is required to create such a serious type of injury [10-12]. Motor vehicle accidents are the leading cause of thoracic spine fracture-dislocation, in fact, motor vehicle accidents are the leading cause of all types of spine fractures and SCIs in developing countries [13, 14]. Our individual, in this case, was a 19-year-old with a severe thoracic spine fracture-dislocation at the T7-T8 level and traumatic spondyloptosis with complete paraplegia as a result of a motor vehicle accident. It is well established that similar injuries should be treated with surgical fixation, aiming to reduce pain, prevent post traumatic spinal deformity and allowing for early rehabilitation [15-19]. This type of injury can be treated surgically by either anterior approach, posterior approach, or combined approach. A systematic review done by Zhu et al., comparing anterior versus posterior approaches in the treatment of thoracolumbar spine fractures, found that a posterior approach had significantly better canal decompression, less blood loss and shorter operative time [20].

Obied et al. reported three cases similar to ours treated by an all posterior approach with total vertebrectomy and spinal shortening in thoracic spine dislocation and complete paraplegia [8]. We decided to avoid an anterior approach in this individual so as to not cause any extra morbidity, particularly to the respiratory system, as the individual had multiple injuries including hemothorax, lung contusion, and DVT was with risk of developing pulmonary embolism with longer operative time. Another advantage of the posterior approach was to minimize blood loss since the individual was on a therapeutic dose of anticoagulation to treat the DVT. A prophylactic inferior vena cava filter was inserted before the operation as the risk of DVT in an individual with a complete SCI is high, and it was removed post-operatively to lower the potential complications of keeping it [21-23].

The primary goal in thoracic spine fracture-dislocation surgery is the correction of the deformity, rigid fixation, and achieving proper bone fusion [7-9]. With the significant overlap at the fracture-dislocation site in this individual, we felt that we could achieve all the goals of the surgery by a posterior approach and vertebrectomy with spinal shortening. Another advantage of this procedure is achieving direct bone contact between the lower endplate of $\mathrm{T} 7$ and the upper endplate of T9, aiming for better fusion and 
Fig. 3 a, b Anteroposterior and lateral radiographs of the thoracic spine, day 1 post operatively with realigned spine.

Fig. 4 a, b Coronal and sagittal cuts of the thoracic spine computed tomography 2 weeks after fixation.
A

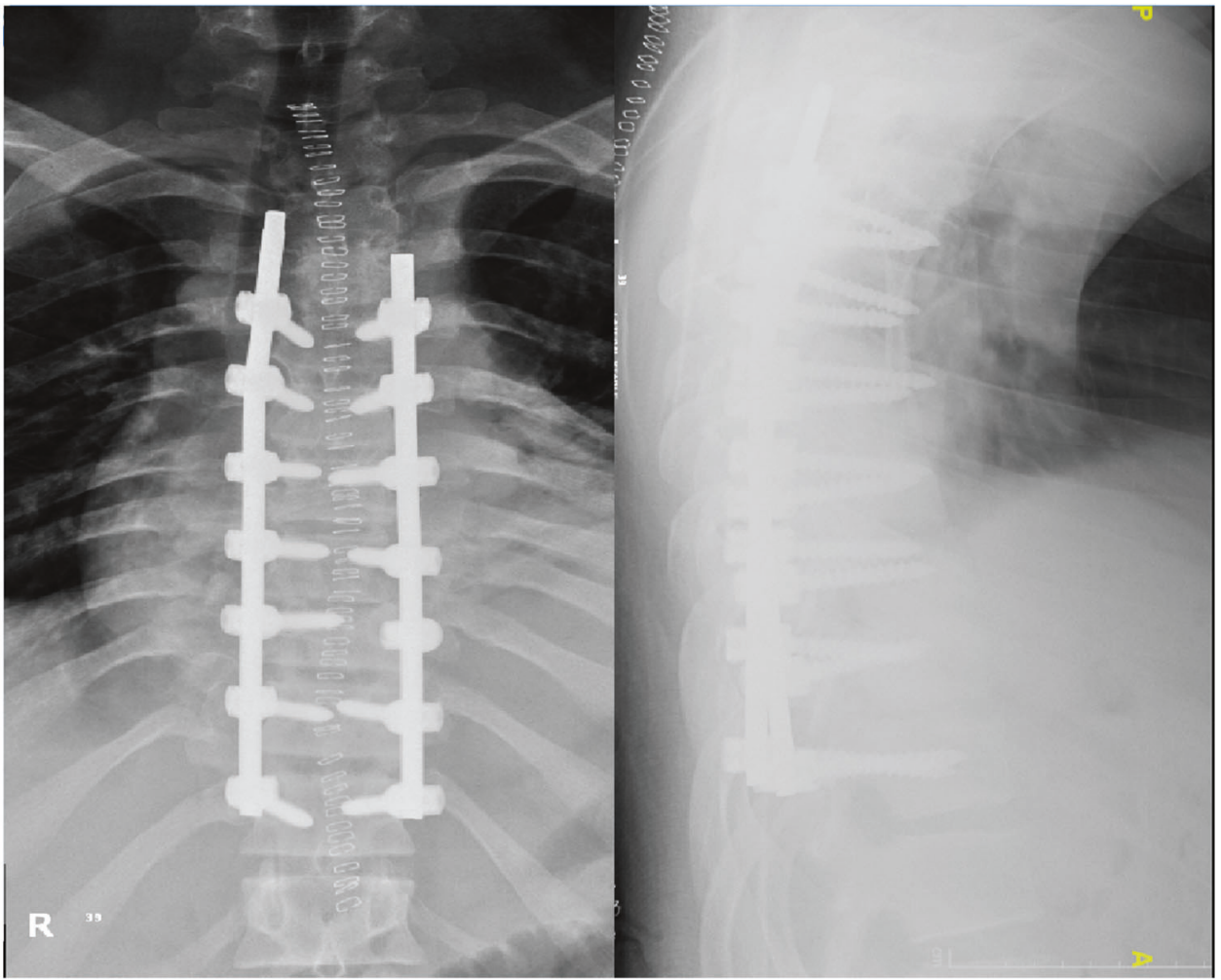

A

B

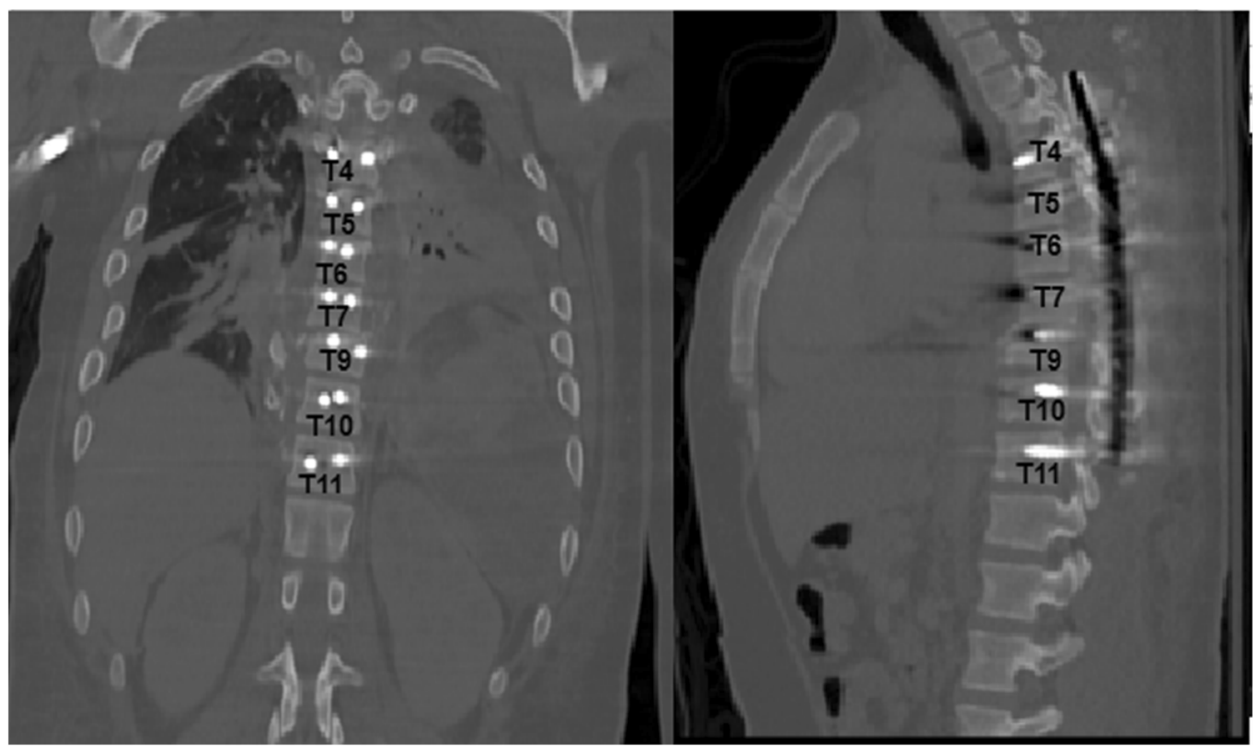

stronger biomechanical construct. Following vertebrectomy, a reduction was achieved by using extended tap pedicle screws (also known as reduction screws) in the proximal segment that is displaced anteriorly (T4-T7) and the reduction was done sequentially to distribute the stress among all the eight screws.
For individuals presenting with significant thoracic fracture-dislocation with severe overlap and complete SCI, we recommend consideration of an all posterior approach, pedicle screws fixation, total vertebrectomy, and spinal shortening to achieve a solid construct with an excellent bone-to-bone contact and deformity correction. 
Fig. 5 a, b Anteroposterior and lateral radiographs of the thoracic spine, 6 months after the surgery showing fused spine in the alignment.
A

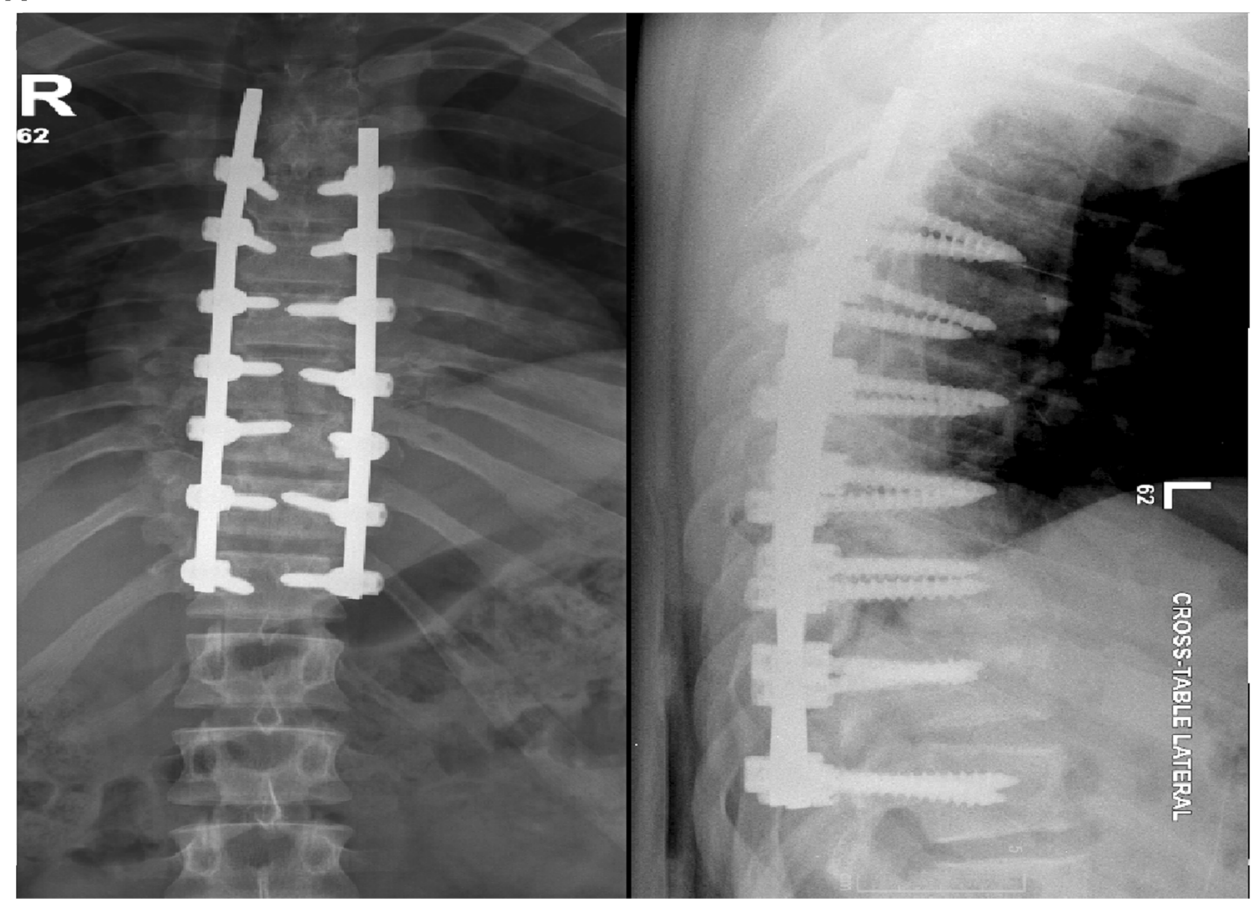

\section{Compliance with ethical standards}

Conflict of interest The authors declare that they have no conflict of interest.

Publisher's note Springer Nature remains neutral with regard to jurisdictional claims in published maps and institutional affiliations.

\section{References}

1. Levine AM. Facet fractures and dislocations of the thoracolumbar spine. In: Levine AM, Eismont FJ, Garfin SR, et al. Editors. Spine trauma. Philadelphia: Saunders; 1998. p. 415-427.

2. Bradford DS, Tribus CB. Vertebral column resection for the treatment of rigid coronal decompensation. Spine. 1997;22: 1590-9. 3

3. MacLennan A. Scoliosis. Br Med J. 1922;2:865-6.

4. Shimizu K, Ido K, Fujio K, Tanaka K, Nakamura T. Total spondylectomy and spinal shortening for giant-cell tumour of spine. Lancet. 1996;348:342.

5. Pappou IP, Papadopoulos EC, Swanson AN, et al. Pott disease in the thoracolumbar spine with marked kyphosis and progressive paraplegia necessitating posterior vertebral column resection and anterior reconstruction with a cage. Spine. 2006;31:E123-E127.

6. Reyes-Sanchez A, Rosales LM, Miramontes VP, et al. Treatment of thoracolumbar burst fractures by vertebral shortening. Eur Spine J. 2002;11:8-12.

7. Barbera J. T12-L1 telescoped chronic dislocation treated by en bloc one-piece spondylectomy and spine shortening. J Spinal Disord Tech. 2004;17:163-166.

8. Obeid I, Guérin P, Gille $\mathrm{O}$, et al. Total vertebrectomy and spine shortening in the management of acute thoracic spine fracture dislocation: technical note and report of 3 cases. J Spinal Disord Tech. 2011;24:340-345.
9. Barcelos A, Botelho R. Treatment of subacute thoracic spine fracture-dislocation by total vertebrectomy and spine shortening. J Neurosurg Spine. 2013;18:194-200.

10. Zhang S, Yan T-B. Severe fracture-dislocation of the thoracic spine without any neurological deficit. World J Surg Oncol. 2017;15:3.

11. Denis F. The three column spine and its significance in the classification of acute thoracolumbar spinal injuries. Spine. 1983;8:817-31.

12. Bohlman HH, Freehafer A, Dejak J. The results of treatment of acute injuries of the upper thoracic spine with paralysis. J Bone Jt Surg Am. 1985;67:360-9.

13. Rahimi-Movaghar V, Sayyah MK, Akbari H, et al. Epidemiology of traumatic spinal cord injury in developing countries: a systematic review. Neuroepidemiology. 2013;41:65-85.

14. Aleissa S, Alassiri S, Aljehani RM, et al. Neurological disability among adults following traumatic spinal fractures in Saudi Arabia: a retrospective single-center medical record review. Ann Saudi Med. 2019;39:8-12.

15. Joaquim AF, Patel AA.Thoracolumbar spine trauma: evaluation and surgical decision making.J Craniovertebr Junction Spine. 2013;4:3-9.

16. Vaccaro AR, Oner C, Kepler CK, et al. AOSpine Spinal Cord Injury \& Trauma Knowledge Forum. AOSpine thoracolumbar spine injury classification system: fracture description, neurological status, and key modifiers. Spine. 2013;38:2028-37.

17. Vaccaro AR, Zeiller SC, Hulbert RJ, et al. The thoracolumbar injury severity score: a proposed treatment algorithm. J Spinal Disord Tech. 2005;18:209-15.

18. Joaquim AF, Ghizoni E, Tedeschi H, et al. Clinical results of patients with thoracolumbar spine trauma treated according to the thoracolumbar injury classification system and severity score. J Neurosurg Spine. 2014;20:562-7.

19. Joaquim AF, Schroeder GD, Patel AA, et al. Clinical and radiological outcome of non-surgical management of thoracic and lumbar spinal fracture-dislocations-a historical analysis in the era of modern spinal surgery. J Spinal Cord Med. 2018;43:3-9. 
20. Zhu Q, Shi F, Cai W, et al. Comparison of anterior versus posterior approach in the treatment of thoracolumbar fractures: a systematic review. Int Surg. 2015;100:1124-33.

21. Grewal S, Chamarthy MR, Kalva SP. Complications of inferior vena cava filters. Cardiovascular Diagnosis Ther. 2016;6:632-41.
22. Mathew J, Povlow MR, Fong A. Get off my back! Inferior vena cava filter erosion into the lumbar spine. BMJ Case Rep. 2019;12.

23. Alabed S, Belci M, Middendorp JJV, et al. Thromboembolism in the sub-acute phase of spinal cord injury: a systematic review of the literature. Asian Spine J. 2016;10:972. 